\title{
Heating up branes in gauged supergravity
}

\section{Kevin Goldstein, Suresh Nampuri and Álvaro Véliz-Osorio}

NITheP, School of Physics and Centre for Theoretical Physics, University of the Witwatersrand, Johannesburg, WITS 2050, South Africa

E-mail: Kevin.Goldstein@wits.ac.za, nampuri@gmail.com, aveliz@gmail.com

ABSTRACT: In this note, we explore the solution space of non-extremal black objects in $4 D$ and $5 D \mathcal{N}=2$ gauged supergravity in the presence of fluxes. We present first order rewritings of the $4 D$ action for a classes of non-extremal dyonic and electric solutions with electric flux backgrounds. Additionally, we obtain the non-extremal version of the Nernst brane in $A d S_{5}$ using a simple deformation. Finally, we develop a new technique to deform extremal black solutions in $4 D$ to non-extremal solutions by an analysis of the symmetries of the equations of motion.

KeYwords: Black Holes in String Theory, AdS-CFT Correspondence

ARXIV EPRINT: 1406.2937 


\section{Contents}

1 Introduction 1

2 Equations of motion and extremal black objects 2

3 First-order flow equations for non-extremal branes 4

3.1 Electric black brane rewriting 5

$\begin{array}{lll}3.2 & \text { Dyonic rewriting } & 10\end{array}$

4 Hot Nernst brane in $A d S_{5} \quad 10$

$\begin{array}{llr}5 & \text { Hot deformations } & 11\end{array}$

$\begin{array}{llr}6 & \text { Conclusions } & 14\end{array}$

$\begin{array}{ll}\text { A Notation } & 14\end{array}$

$\begin{array}{ll}\text { B Explicit gauge field ansatz } & 16\end{array}$

\section{Introduction}

Black holes offer excellent laboratories for testing the predictions of any purported quantum theory of gravity such as string theory. In particular, one of the prime objectives of any such theory is to provide a microscopic understanding of the Hilbert space of these objects and thereby arrive at a first principle derivation of their thermodynamic properties which can be written down heuristically in Einstein's gravity. Furthermore, under gauge-gravity duality, black objects are presumably holographically dual to thermal ensembles in a field theory. If this is the case, at strong coupling, field theoretic phenomena in the presence of such an thermal ensemble, can be modeled in terms of the physics of matter in the black background. In particular, black solutions which obey the third law of thermodynamics, having vanishing entropy at zero temperature (Nernst solutions), could be very useful in understanding quantum critical phase transitions in the dual field theory. Hence, developing and exploring the solution spaces of black objects in actions arising as low-energy limits of string theory - namely certain supergravities (SUGRA) - is of paramount import in understanding non-perturbative aspects of string theory and potentially, strongly coupled field theories.

The equations of motion (EOMs) of these supergravity actions are second-order nonlinear and hence enormously non-trivial to solve. For a special class of extremal black solutions in $4 D$ and $5 D \mathcal{N}=2$ gauged supergravity, considerable advances have been made in the last half-decade (see cf. $[2,3,8]$ and [10]). These solutions are amenable to 
a first order sum-of-squared-terms rewriting, which simplifies the problem to solving first order equations. A natural generalization is to develop techniques to study non-extremal black solutions. In particular, given the fact that a large class of extremal solutions are known, we investigate methods of generating non-extremal solutions from extremal ones which could be of great practical value.

Specifically, after a brief review of the action and the $4 D$ EOMs in section 2, we develop two first order first-order rewritings of this action for a class of non-extremal electric and dyonic black states with electric fluxes, in section 3. In section 4, we deform the parameter space of a class of extremal black solutions in asymptotically $A d S_{5}$ spaces $\left(A A d S_{5}\right)$ to obtain the non-extremal equivalent of a zero-entropy black Nernst brane in the same space. In section 5 , we analyze the second order EOMs in $4 D$ to identify certain symmetries. We exploit these symmetries to compensate non-extremal deformations of the metric with transformations of the parameters of the solution. The techniques developed in each section are illustrated by generating example black solutions. Our formal results are applicable to black solutions with flat, spherical or hyperbolic horizons. Finally in the conclusion we discuss possible extensions and applications to $5 D$.

\section{Equations of motion and extremal black objects}

The main purpose of this note is to study black gravitational solutions of $\mathcal{N}=2$ gauged supergravity. We consider Lagrangians of the form

$$
S=\int d x^{4} \sqrt{-g}\left(R-2 g_{i \bar{\jmath}} \partial_{\mu} z^{i} \partial^{\mu} \bar{z}^{j}-f_{I J} F_{\mu \nu}^{I} F^{J \mu \nu}-\frac{1}{2} \tilde{f}_{I J} F_{\mu \nu}^{I} F_{\rho \sigma}^{J} \epsilon^{\mu \nu \rho \sigma}-2 V_{g}(z, \bar{z})\right),
$$

where the $z^{i}$ are complex scalar fields, and the $F^{I}$ are abelian gauge fields. The structure of the different pieces of the Lagrangian - namely $f_{I J}, g_{i \bar{\jmath}}$ and $V_{g}(z, \bar{z})$ - and the range of the indices $(i, I)$, can be found from very specific prescriptions (see appendix A), but for the sake of generality, we make no assumptions at present.

For the action (2.1), Einstein's equations read

$$
R_{\mu \nu}-2 g_{i \bar{\jmath}} \partial_{\mu} z^{i} \partial_{\nu} \bar{z}^{j}=f_{I J}\left(2 F_{\mu \lambda}^{I} F_{\nu}^{J \lambda}-\frac{1}{2} g_{\mu \nu} F_{\kappa \lambda}^{I} F^{J \kappa \lambda}\right)+g_{\mu \nu} V_{g}(z, \bar{z})
$$

As we are interested in studying static solutions with purely radial dependence, we take $z^{i}=z^{i}(r)$, assume the line element is of the form

$$
d s^{2}=-e^{2 \alpha(r)} d t^{2}+e^{-2 \alpha(r)} d r^{2}+e^{2 \beta(r)} d \Omega_{k}^{2},
$$

with our ansatze for the gauge fields given explicitly in appendix B. The label $k$ in $(2.3)$ denotes the assumed metric of the transverse spacial foliation with $k=-1,0,1$ standing for hyperbolic, flat and spherical respectively. We will also use the alternative notation

$$
a(r)=e^{\alpha(r)} \quad b(r)=e^{\beta(r)} \quad a(r) b(r)=e^{\psi(r)}
$$

for the warp factors whenever this makes the expressions more compact. 
Given the assumptions mentioned above, Einstein's equations become

$$
\left(a^{2} b^{2}\right)^{\prime \prime}=2\left(k-2 b^{2} V_{g}\right),
$$

and

$$
\frac{b^{\prime \prime}}{b}=-g_{i \bar{\jmath}} \partial_{r} z^{i} \partial_{r} \bar{z}^{j}
$$

where " ' " denotes derivatives with respect to $r$. In addition to these, the following first-order constraint must be fulfilled

$$
-\frac{1}{2}\left(a^{2}\left(b^{2}\right)^{\prime}\right)^{\prime}=\frac{V_{b}}{b^{2}}+b^{2} V_{g}-k .
$$

In the previous expressions we introduced the potential

$$
V_{b}=f^{I J}\left(Q_{I}-\tilde{f}_{I K} P^{K}\right)\left(Q_{J}-\tilde{f}_{J K} P^{K}\right)+f_{I J} P^{I} P^{J}
$$

with $f^{I J}$ the inverse of $f_{J K}$. This potential encodes all the contributions coming from the gauge field's electric $Q_{I}$ and magnetic $P^{I}$ charges (see appendix B). In addition to $(2.5),(2.6)$ and $(2.7)$ we have the equations of motion for the scalars:

$$
\partial_{r}\left(a^{2} b^{2} g_{k \bar{\jmath}} \partial_{r} \bar{z}^{j}\right)=a^{2} b^{2} \partial_{k}\left(g_{i \bar{\jmath}}\right) \partial_{r} z^{i} \partial_{r} \bar{z}^{j}+\frac{\partial_{k} V_{b}}{b^{2}}+b^{2} \partial_{k} V_{g}
$$

Clearly, it is rather complicated to solve the equations above in general. There exist, however, powerful techniques that are applicable for certain subclasses of solutions such as extremal black solutions and BPS black states. In the following we will concentrate mainly on solutions which are asymptotically $A d S_{4} \cdot{ }^{1}$

Let us start by discussing extremal black solutions in $A d S_{4}$, these are solutions whose near-horizon geometry is described by an $A d S_{2} \times \Sigma_{k}$ metric, where $\Sigma_{1}=S^{2}$ and $\Sigma_{0}=\mathbb{R}^{2}$. On this kind of backgrounds, $b(r)$ takes up a constant value, denoted by $\sigma$, encoding the entropy of the solution while for the other warp factor we have

$$
a=\frac{r}{l_{2}},
$$

where $l_{2}$ corresponds to the $A d S_{2}$ radius. Inserting this geometry into (2.6) and (2.7) we find that the scalars become constants whose values are determined by

$$
\partial_{k} V_{b}=-\sigma^{4} \partial_{k} V_{g}
$$

Therefore they depend only on the values of the fluxes and charges of the system. ${ }^{2}$ Meanwhile, the two parameters that specify the metric can be extracted from the algebraic relations

$$
k=\frac{V_{b}}{\sigma^{2}}+\sigma^{2} V_{g} \quad\left(\frac{\sigma}{l_{2}}\right)^{2}=\frac{V_{b}}{\sigma^{2}}-\sigma^{2} V_{g}
$$

\footnotetext{
${ }^{1}$ These solutions are specially relevant for $A d S_{4} / C F T_{3}$ holography.

${ }^{2}$ This is the well-known attractor mechanism (see cf. [12, 14, 21])
} 
derived from (2.5) and (2.6). For the case of black branes, i.e. solutions with flat horizon topology $(k=0)$ the equations $(2.11)$ yield the simple relations

$$
l_{2}^{2}=-\frac{1}{2 V_{g}} \quad \sigma^{4}=-\frac{V_{b}}{V_{g}},
$$

which relate the geometric parameters to the potentials in a transparent way.

We turn our attention now to the asymptotic behavior. We want the metric to approach $A d S_{4}$ at ifinity i.e.

$$
a \stackrel{r \rightarrow \infty}{\longrightarrow} b \stackrel{r \rightarrow \infty}{\longrightarrow} \frac{r}{l_{4}} .
$$

Once again by inserting this into the equations of motion, we find that the scalars take up constant values which now are determined by

$$
\partial_{k} V_{g}=0
$$

in other words, the scalar fields must flow towards critical points of the $V_{g}$ potential at infinity. While the only geometric parameter, the AdS radius, reads

$$
l_{4}^{2}=-\frac{3}{V_{g}} .
$$

Therefore, if for certain given quantum numbers we wish to find an extremal black hole/brane, first we need to show that both (2.11) and (2.15) have compatible solutions that give rise to sensible physical parameters (e.g. positive AdS-radii) and then we must find solutions of the equations of motion $a(r), b(r)$ and $z^{i}(r)$ that interpolate between the two AdS geometries.

The procedure described above is in general challenging, but in the context of $\mathcal{N}=2$ gauged supergravity, there is a large class of extremal black branes that can be described by a simpler set of first order equations equations $[3,8]$. As a matter of fact, interpolating solutions corresponding to these equations are characterized by the relation

$$
a^{2} b^{2}=r\left(\frac{r}{l_{4}^{2}}+\frac{\sigma}{l_{2}}\right)
$$

with the scalar field solutions constructed as combinations of harmonic functions $([16,17])$.

\section{First-order flow equations for non-extremal branes}

As mentioned at the end of the previous section, being able to provide a first order description for the system of interest makes the problem of finding solutions much more tractable. Given that for $\mathcal{N}=2$ gauged supergravity models, extremal configurations can be described by first-order equations, it is natural to wonder whether non-extremal configurations can be found in a similar fashion. Fortunately it is in fact possible to find first order descriptions for non-extremal configurations ([2, 3, 13] and [6]). Unfortunately, these descriptions are generically non-trivial to construct and can be set up only for certain subclasses of black objects. In the following, we construct two new first-order rewritings for non-extremal black branes: one for generating classes of electric black branes and the other for dyonic solutions. 


\subsection{Electric black brane rewriting}

Consider the line element,

$$
d x^{2}=-e^{2 \alpha(r)} d t^{2}+e^{2 \nu(r)} d r^{2}+e^{2 \beta(r)}\left(d x^{2}+d y^{2}\right) .
$$

The first rewriting that we find in this section is valid for solutions supported by electric charges and fluxes. Inserting the line element (3.1) and our gauge ansatz (appendix B) into the $4 D \mathcal{N}=2$ gauged supergravity action (2.1), yields the one-dimensional effective lagrangian density

$$
\mathcal{L}=e^{2 \beta+\alpha-\nu}\left(\left(\beta^{\prime}\right)^{2}+2 \beta^{\prime} \alpha^{\prime}-N_{I J}\left(X^{I}\right)^{\prime}\left(\bar{X}^{J}\right)^{\prime}-e^{2 \nu} V_{\text {tot }}(X, \bar{X})\right),
$$

where

$$
V_{\mathrm{tot}}=g^{2} V_{g}+e^{-4 \beta} V_{b},
$$

$N_{I J}$ is given by (A.5) and the scalars $X$ are related to the scalars $z$ by (A.2), (A.4) Here, the constant $g^{2}$ is inserted to keep track of terms arising from the flux potential, $V_{g}$. But, henceforth, we will formally absorb the constant into the definition of the flux potential by rescaling the flux quantum numbers as $h_{I} \rightarrow h_{I} / g$, so as to be consistent with notation in literature.

As a first step, we decompose the warp factors $\alpha$ and $\nu$ appearing in the lineelement (3.1) into

$$
\alpha=\alpha_{0}+\frac{1}{2} \ln f \quad \nu=\nu_{0}-\frac{1}{2} \ln f,
$$

where

$$
f=-\mu r+g^{2} e^{2 \alpha_{1}(r)} .
$$

The horizon will correspond to physical solutions of the equation

$$
\mu r=g^{2} e^{2 \alpha_{1}(r)}
$$

As we will see in some examples later, by changing the value of $\mu$ we modify the root structure of this equation so that the constant parameter $\mu$ parametrizes the non-extremality of the solution. To proceed, we decompose $\mathcal{L}$ into powers of $g^{2}$,

$$
\mathcal{L}=\mathcal{L}_{0}+g^{2} \mathcal{L}_{2}
$$

The next step is to rewrite both $\mathcal{L}_{0}$ and $\mathcal{L}_{2}$ in terms of squares of first-order flow equations. From this procedure, we will obtain an overdetermined system whose consistency must be checked. This rewriting is analogous to the one performed in five-dimensional $\mathcal{N}=2, \mathrm{U}(1)$ gauged supergravity in $[2,6]$. Let us start with $\mathcal{L}_{0}$. For the sake of simplicity, we use the diffeomorphism invariance of the theory, changing the radial coordinate, to set $\nu_{0}=2 \beta+\alpha_{0}$ and obtain

$$
\mathcal{L}_{0}=-\mu \beta^{\prime}-\mu r\left(\left(\beta^{\prime}\right)^{2}+2 \beta^{\prime} \alpha_{0}^{\prime}-N_{I J}\left(X^{I}\right)^{\prime}\left(\bar{X}^{J}\right)^{\prime}\right)-e^{2 \alpha_{0}} \mathcal{G}^{I J} Q_{I} Q_{J} .
$$

Next, we introduce the combination

$$
\mathcal{E}^{I}=X^{I}-\alpha_{0}^{\prime} X^{I}+e^{\alpha_{0}} N^{I J} \lambda_{J}
$$


where $\lambda_{J}$ are real parameters that are a priori arbitrary. This expression is the candidate for the first order equation driving the scalar fields. Using (A.4) we get

$$
\bar{X}^{I} N_{I J} \mathcal{E}^{J}=\bar{X}^{I} N_{I J} X^{J}+\alpha_{0}^{\prime}+e^{\alpha_{0}} \lambda_{I} \bar{X}^{I} .
$$

Inserting the above equation into $\mathcal{L}_{0}$ we find

$$
\begin{aligned}
\mathcal{L}_{0}= & -\mu \beta^{\prime}-\mu \lambda_{I}\left[r e^{\alpha_{0}}\left(X^{I}+\bar{X}^{I}\right)\right]^{\prime} \\
& -\mu r\left[\left(\beta^{\prime}+\alpha_{0}^{\prime}\right)^{2}-\mathcal{G}^{I J} N_{I P} \mathcal{E}^{P} N_{J Q} \overline{\mathcal{E}}^{Q}+2 \bar{X}^{I} N_{I J} \mathcal{E}^{J} X^{K} N_{K L} \overline{\mathcal{E}}^{L}\right. \\
& \left.-2\left(\alpha_{0}^{\prime}+e^{\alpha_{0}} \lambda_{I} X^{I}\right)\left(\alpha_{0}^{\prime}+e^{\alpha_{0}} \lambda_{I} \bar{X}^{I}\right)\right] \\
& +\mu e^{\alpha_{0}} \lambda_{I}\left(X^{I}+\bar{X}^{I}\right)-e^{2 \alpha_{0}} \mathcal{G}^{I J}\left(r \mu \lambda_{I} \lambda_{J}+Q_{I} Q_{J}\right)
\end{aligned}
$$

where we introduced the positive definite matrix

$$
\mathcal{G}^{I J}=N^{I J}+2 e^{K} \bar{X}^{I} X^{J}
$$

defined on the scalar moduli space with the Kähler potential, $K$, given by (A.7). Notice that the first line of (3.11) contains total derivatives, while the second and third lines contain squares of various combinations of fields. The last line encodes additional constraints that will be discussed soon.

Requiring the variation of the second and third lines of $\mathcal{L}_{0}$ with respect to the fields $\left(\beta, \alpha_{0}, X^{I}\right)$ to vanish can be achieved by setting the various squares to zero. This yields $X^{I}=\bar{X}^{I}$ as well as the first-order flow equations

$$
\begin{aligned}
& \beta^{\prime}=-\alpha_{0}^{\prime}, \\
& \mathcal{E}^{I}=0, \\
& \alpha_{0}^{\prime}=-e^{\alpha_{0}} \lambda_{I} X^{I} .
\end{aligned}
$$

These can be readily integrated by introducing the rescaled scalar fields

$$
Y^{I}=e^{-\alpha_{0}} X^{I}
$$

giving

$$
\begin{aligned}
e^{2 \beta} & =e^{-2 \alpha_{0}}=Y^{I} H_{I}, \\
Y^{I} & =-N^{I J} H_{J},
\end{aligned}
$$

where $H_{I}=\gamma_{I}+\lambda_{I} r$, with constant $\gamma_{I}$. To obtain (3.15) we used the relations $F_{I J K} X^{K}=$ 0 (A.1) and $\left(X^{I} N_{I J} \bar{X}^{J}\right)^{\prime}=0$ (A.4), as well as the fact that the $X^{I}$ 's are real.

To conclude the $\mathcal{L}_{0}$ rewriting, we must analyze the variation of the terms in the last line of (3.11). Their variation should result in equations that are consistent with the flow equations (3.13). To this end, we restrict the analysis to models in which satisfy two simplifying conditions. First, we demand that there are no linear terms proportional to $(X-\bar{X})^{K}$ in $\mathcal{G}^{I J}$, i.e.

$$
\mathcal{G}^{I J}(X, \bar{X})=\mathcal{G}^{I J}(\operatorname{Re} X, \operatorname{Re} X)+\mathcal{O}\left((X-\bar{X})^{2}\right),
$$


so that we may solve the variational equations steming from the last line of (3.11) by setting $(X-\bar{X})^{I}=0$. Second, we assume that the physical charges $Q_{I}$ can be expressed in terms of the constant vectors $\lambda_{I}$ and $\gamma_{I}$ by using the relation

$$
\mathcal{G}^{I J}(\operatorname{Re} X, \operatorname{Re} X) Q_{I} Q_{J}=\mu \mathcal{G}^{I J}(\operatorname{Re} X, \operatorname{Re} X) \lambda_{I} \gamma_{J} .
$$

Generically, this can be achieved in models in which $\mathcal{G}^{I J}(\operatorname{Re} X, \operatorname{Re} X)$ is diagonal or off-diagonal.

Imposing the relation $(X-\bar{X})^{I}=0$ as well as (3.17), the last line of (3.11) becomes

$$
\mu e^{\alpha_{0}}\left(2 \lambda_{I} x^{I}-e^{\alpha_{0}} \mathcal{G}^{I J}(x, x) H_{I} \lambda_{J}\right),
$$

where $x^{I}=\operatorname{Re} X^{I}$. Denoting the combination (3.18) by $\Delta$, we compute its variation with respect to $\alpha_{0}$ and with respect to the scalar fields $x^{I}$, respectively, and obtain

$$
\begin{gathered}
\mu \delta\left(e^{\alpha_{0}}\right)\left[2 \lambda_{I} x^{I}-e^{\alpha_{0}} \mathcal{G}^{I J}(x, x) H_{I} \lambda_{J}\right]=0, \\
\mu e^{\alpha_{0}} \delta x^{L}\left[2 \lambda_{L}-i e^{\alpha_{0}} N^{I P}\left(F_{P Q L}-\bar{F}_{\bar{P} \bar{Q} \bar{L}}\right) N^{Q J} H_{I} \lambda_{J}\right. \\
\left.-2 e^{\alpha_{0}}\left(\left(x^{I} H_{I}\right) \lambda_{L}+\left(x^{I} \lambda_{I}\right) H_{L}\right)\right]=0,
\end{gathered}
$$

where in the second equation the quantities $N^{I J}$ and $F_{I J K}$ are evaluated at $x^{L}$. Using (3.15), we find that the first equation of (3.19) is satisfied, while for the second equation we obtain

$$
\mu e^{\alpha_{0}}\left(x^{I} \lambda_{I}\right) \delta x^{L} N_{L K} x^{K}=0
$$

Recalling that the variation of (A.4) yields $\delta X^{I} N_{I J} \bar{X}^{J}+X^{I} N_{I J} \delta \bar{X}^{J}=0$, and setting $X^{I}=\bar{X}^{I}$, gives $\delta x^{I} N_{I J} x^{J}=0$. This equals (3.20), hence we conclude that for a model satisfying (3.17) the conditions (3.19) stemming from the variation of $\Delta$ are satisfied by a solution to the first-order flow equations (3.13).

Now we turn our attention to $\mathcal{L}_{2}$, which is given by

$$
\mathcal{L}_{2}=e^{2 \alpha_{1}}\left(\left(\beta^{\prime}\right)^{2}+2 \beta^{\prime}\left(\alpha_{0}^{\prime}+\alpha_{1}^{\prime}\right)-N_{I J}\left(X^{I}\right)^{\prime}\left(\bar{X}^{J}\right)^{\prime}-e^{4 \beta+2 \alpha_{0}-2 \alpha_{1}} V_{g}(X, \bar{X})\right) .
$$

Following [2], we rewrite this as

$$
\begin{gathered}
\mathcal{L}_{2}=e^{2 \alpha_{1}}\left\{\left(\beta^{\prime}+\alpha_{0}^{\prime}+\alpha_{1}^{\prime}+2 \alpha e^{2 A+\alpha_{0}-\alpha_{1}} h_{I} X^{I}\right)\left(\beta^{\prime}+\alpha_{0}^{\prime}+\alpha_{1}^{\prime}+2 \alpha e^{2 \beta+\alpha_{0}-\alpha_{1}} h_{I} \bar{X}^{I}\right)\right. \\
-\left(\alpha_{0}^{\prime}+\alpha_{1}^{\prime}+\alpha e^{2 \beta+\alpha_{0}-\alpha_{1}} h_{I} X^{I}\right)\left(\alpha_{0}^{\prime}+\alpha_{1}^{\prime}+\alpha e^{2 \beta+\alpha_{0}-\alpha_{1}} h_{I} \bar{X}^{I}\right) \\
-\left(\beta^{\prime}+\alpha e^{2 \beta+\alpha_{0}-\alpha_{1}} h_{I} X^{I}\right)\left(\beta^{\prime}+\alpha e^{2 \beta+\alpha_{0}-\alpha_{1}} h_{I} \bar{X}^{I}\right) \\
-N_{I J}\left(\left(X^{I}\right)^{\prime}+\beta^{\prime} X^{I}-\kappa e^{2 \beta+\alpha_{0}-\alpha_{1}} N^{I K} h_{K}\right) \times \\
\left.\times\left(\left(\bar{X}^{J}\right)^{\prime}+\beta^{\prime} \bar{X}^{J}-\kappa e^{2 \beta+\alpha_{0}-\alpha_{1}} N^{J L} h_{L}\right)\right\} \\
-\kappa\left[e^{2 \beta+\alpha_{0}+\alpha_{1}} h_{I}\left(X^{I}+\bar{X}^{I}\right)\right]^{\prime},
\end{gathered}
$$

where $\kappa= \pm 1$ (corresponding to two possible rewritings). This expresses $\mathcal{L}_{2}$ in terms of squares of combinations of fields. Setting these squares to zero ensures the vanishing of 
the variation of $\mathcal{L}_{2}$ with respect to the various fields. This yields $X^{I}=\bar{X}^{I}$ as well as the first-order flow equations ${ }^{3}$

$$
\begin{aligned}
\beta^{\prime}+\alpha_{0}^{\prime}+\alpha_{1}^{\prime} & =-2 \kappa e^{2 \beta+\alpha_{0}-\alpha_{1}} h_{I} X^{I}, \\
\alpha_{0}^{\prime}+\kappa_{1}^{\prime} & =-\kappa e^{2 \beta+\alpha_{0}-\alpha_{1}} h_{I} X^{I}, \\
\beta^{\prime} & =-\kappa e^{2 \beta+\alpha_{0}-\alpha_{1}} h_{I} X^{I} . \\
\left(X^{I}\right)^{\prime}+\beta^{\prime} X^{I} & =\kappa e^{2 \beta+\alpha_{0}-\alpha_{1}} N^{I K} h_{K} .
\end{aligned}
$$

By demanding compatibility with the first-order flow equations (3.13) obtained from $\mathcal{L}_{0}$ we find

$$
2 \alpha_{0}+\alpha_{1}=0
$$

as well as

$$
\lambda_{I}=-\kappa h_{I}
$$

The harmonic functions $H_{I}$ entering in the solution (3.15) are thus given by

$$
H_{I}=\gamma_{I}-\kappa h_{I} r
$$

Summarizing, we have obtained a non-extremal static electrically charged black brane solutions are described by

$$
\begin{aligned}
d s^{2} & =-e^{-2 \beta} f d t^{2}+e^{2 \beta} f^{-1} d r^{2}+e^{2 \beta}\left(d x^{2}+d y^{2}\right), \\
f & =-\mu r+g^{2} e^{4 \beta}, \\
e^{2 \beta} & =Y^{I} H_{I} \\
Y^{I} & =\bar{Y}^{I}=-N^{I J} H_{J}, \\
H_{I} & =\gamma_{I}-\kappa h_{I} r \quad, \quad \kappa= \pm 1,
\end{aligned}
$$

where the constants $\gamma_{I}$ are related to the physical charges $Q_{I}$ by

$$
\mathcal{G}^{I J} Q_{I} Q_{J}=-\kappa \mu \mathcal{G}^{I J} h_{I} \gamma_{J}
$$

In the following we discuss two examples. First, we consider an $\mathcal{N}=2$ model based on the prepotential

$$
F(X)=-2 i \sqrt{X^{0} X^{1} X^{2} X^{3}} .
$$

This model comes from the $\mathrm{U}(1)^{4}$ truncation of $\mathcal{N}=8$ gauged supergravity in four dimensions [9]. Using (3.30), we compute $N_{I J}$ for this prepotential using and find

$$
N_{I J}=\frac{i}{2}\left(2 \delta_{I J}-1\right) \frac{F(X)}{X^{I} X^{J}}
$$

Hence (3.12) reads

$$
\mathcal{G}^{I J}=\frac{1}{4} X^{I} X^{J}\left(8+(-1)^{\delta_{I J}} \frac{2 i}{F(X)}\right)
$$

\footnotetext{
${ }^{3} \mathrm{~A}$ first order rewriting for non-extremal black solutions, with a different re-parametrization was independently developed in [13].
} 
Inserting (3.30) into (A.4) yields

$$
F(X)=-\frac{i}{4}
$$

therefore

$$
\mathcal{G}^{I J}=4\left(X^{I}\right)^{2} \delta^{I J}
$$

as well as

$$
e^{2 \beta}=4 i F(Y)=8 \sqrt{Y^{0} Y^{1} Y^{2} Y^{3}} .
$$

Using (3.30) we compute

$$
N_{I J} Y^{J}=-i \frac{F(Y)}{Y^{I}}
$$

and hence

$$
Y^{I}=\frac{1}{4} \frac{e^{2 \beta}}{H_{I}}
$$

where we made use of the third equation of (3.27). Explicitly, the harmonic functions are given by

$$
H_{I}=\frac{1}{h_{I}}\left(\mu^{-1} Q_{I}^{2}+h_{I}^{2} r\right) .
$$

Inserting this into (3.34) gives the warp factor

$$
e^{2 \beta}=2 \sqrt{H_{0} H_{1} H_{2} H_{3}} .
$$

These solutions correspond to the brane solutions in [7]. They interpolate between an $A d S_{4}$ asymptopia with

$$
l_{4}^{2} \sim \frac{1}{\sqrt{h_{0} h_{1} h_{2} h_{3}}}
$$

and the outer horizon given by the largest root of (3.6). It is possible to tune $\mu$ to make the solution extremal by demanding that the following holds at horizon

$$
-\frac{\mu}{4}=g^{2} \beta^{\prime} e^{4 \beta} .
$$

As a second example consider the model based on the prepotential

$$
F(X)=-X^{0} X^{1} .
$$

For this prepotential we have

$$
N_{I J}=-2\left|\epsilon_{I J}\right|
$$

For axion-free configurations, (3.12) is simply

$$
\mathcal{G}^{I J}=\frac{1}{2}\left(\begin{array}{cc}
z^{-1} & 0 \\
0 & z
\end{array}\right),
$$

where we introduced

$$
z=\frac{X^{1}}{X^{0}}
$$

Using (A.4), we find

$$
e^{2 \beta}=4 Y^{0} Y^{1} .
$$


While the scalars are given by

$$
Y^{J}=\frac{1}{2}\left|\epsilon^{I J}\right| H_{J}
$$

Once again, we have a solution that interpolates between $A d S_{4}$ with radius

$$
l_{4}^{2} \sim \frac{1}{\left|h_{0} h_{1}\right|}
$$

and the corresponding horizon.

\subsection{Dyonic rewriting}

Here we develop a first order rewriting to generate a class of dyonic non-extremal solutions in the presence of electric fluxes with $h_{0}=P^{0}=0$. For this set of quantum numbers, the 1D Lagrangian density can be rewritten as a sum of perfect squares which yield the following first order equations:

$$
\begin{aligned}
\left(z^{i}\right)^{\prime} & =\frac{1}{2} g^{i j}\left(\gamma_{j}-2 r \tilde{q}_{j}\right) e^{-\frac{8}{3} \beta} \\
\beta^{\prime} & =\left(-\frac{1}{4} z^{i} h_{i}+\frac{1}{2} g_{i j} z^{i} P^{j}\right) e^{-\alpha_{1}} \\
\alpha_{1}^{\prime} & =2 \beta^{\prime}+2 Q_{0} e^{-2 \beta-\alpha_{1}}
\end{aligned}
$$

with constraints given by:

$$
\begin{gathered}
h_{i} P^{i}=0 \quad-2 Q_{0}=\gamma_{i} P^{i} \quad g^{i j} q_{i} q_{j}=-4 \mu g^{i j} \tilde{q}_{i} \gamma_{j} \\
\frac{1}{3} e^{-2 \beta} z^{i} \tilde{q}_{i}+\frac{1}{3} z^{i} h_{i} e^{-\alpha_{1}}-\frac{2}{3} g_{i j} z_{i} P^{j} e^{-\frac{4}{3} \beta-\alpha_{1}}
\end{gathered}
$$

Notable solutions of the above equations for $Q_{0}=0$ contain the $\eta$-geometries discussed in [11] with relevance to holographic condensed matter issues. Solutions of these equations can be up-lifted to $5 D$ and be shown to be solutions of the first order equations derived from a re-writing of the 5D gauged SUGRA action for the same set of quantum numbers, written in $5 D$ language.

\section{Hot Nernst brane in $A d S_{5}$}

In this section we use first order rewritings for $\mathcal{N}=25 \mathrm{D}$ gauged supergravity [2] to discover and write down a new class of black solutions in $A A d S_{5}$ spaces which in the extremal limit have vanishing entropy. In the extremal limit these satisfy the third law of thermodynamics or the Nernst law and hence, go by the name of Nernst branes. Studying the physics of matter in near-extremal solutions of the Nernst type will potentially shed valuable light on the physics of phase transitions at zero-temperature in the dual $4 D$ field theory, and the phase diagram that captures aspects of this transition as one approaches absolute zero. We will look for these solutions by extremizing the low-energy $5 D \mathcal{N}=2$ gauged supergravity action arising from the compactification of M-theory on a $C Y_{3}$. This action is amenable to first order rewritings for dyonic black solutions [2] in the presence of electric fluxes and 
which are charged under one of the Cartans of the angular momentum group in $5 D$. One can solve the resulting first order equations for a system with no dyonic charges and only an angular momentum $J$ and with electric fluxes $h_{1}, h_{2}$ and $h_{3}$ in the STU model to obtain a family of solutions (see appendix $\mathrm{C}$ in [2]) wherein the scalars, $X^{A},(A=1,2,3)$, are constant through out and the metric is given by

$$
d s^{2}=-e^{2 U} d t^{2}+\frac{d \tau^{2}}{\tau^{2}}+e^{2 B}\left(d x^{2}+d y^{2}\right)+e^{2 W}(d z+C d t)^{2}
$$

and where

$$
\begin{aligned}
e^{B} & =\tau \\
e^{2 W} & =\left(\alpha+\gamma \tau^{4}\right) / \tau^{2} \\
e^{2 U} & =\tau^{6} /\left(\alpha+\gamma \tau^{4}\right) \\
C & =\tau^{4} /\left(\alpha+\gamma \tau^{4}\right)
\end{aligned}
$$

Here $\alpha=\frac{3 J}{4 h_{A} X^{A}}$ is a positive constant, while $\gamma$ is a constant of integration. The above solution is a zero-entropy $A A d S_{5}$ extremal Nernst solution, which can be compactified to $4 D$ and lies in the BPS class of solutions of the $4 D$ rewriting, as observed in [2].

The Nernst brane, being at zero entropy, has no discernible scale in its near horizon geometry. A hot Nernst brane on the other hand would have a natural scale, arising from the temperature. In order to heat up a zero-scale system to a finite scale one, we take a hint from the observation that massive BTZ black holes in $A d S_{3}$ can be thought of as deformations of the angular momentum from static $M=0$ BTZ black holes. Hence we perform a deformation of the $g_{t z}$ term in the above solution via the shift:

$$
C \rightarrow C+\lambda
$$

One can check that the above solution satisfies the EOMs.

In particular, for $\lambda=-\gamma<0$, we observe that the solution has two horizons with the outer horizon given by the zero of $g_{00}=-e^{2 U}+e^{2 W} C^{2}$ at $\tau=(\alpha|\lambda|)^{1 / 4}$, and where the temperature, computed by demanding smoothness of the Euclidean near-horizon Rindler metric is

$$
T=\frac{2 \pi}{|\lambda| \alpha(\alpha \lambda)^{1 / 4}}
$$

The black solutions so obtained, interpolate between the non-extremal Rindler geometry near the horizon of the black brane to $A d S_{5}$. Hence, we have discovered here a family of hot black branes in AAdS which obeys the third law of thermodynamics at zero temperature.

\section{Hot deformations}

So far, we have written down rewritings which allow us to obtain hot black solutions by solving first order equations. However, these rewritings have various constraints on charges and fluxes and it is not possible to always obtain a non-extremal solution for a generic set of charges and fluxes whose extremal counterparts are fully known. The Nernst brane is a 
case in point. So far, there exists no first order re-writing for its non-extremal counterpart. We wrote down the hot solution by performing a deformation on the extremal solution to obtain a new solution of the EOMs.

In this section, we generalize this principle by developing a deformation algorithm for a large class of extremal solutions, that will automatically generate the corresponding non-extremal solutions.

We begin with an outline of the working philosophy behind the deformation of extremal dyonic solutions in $4 D \mathcal{N}=2$ gauged SUGRA, in the presence of fluxes, to non-extremal solutions. In order to generate new solutions of the second order EOMs, we identify deformations that are symmetries of the EOMs. The deformations are implemented in three steps. First, notice that the deformations

$$
\begin{aligned}
(a b)^{2} & \rightarrow(a b)^{2}-\mu r \\
b & \rightarrow b
\end{aligned}
$$

form a symmetry of the equations of motion (2.5) and (2.6). We can promote this transformation to a symmetry of the full set of equations of motion by considering a transformation for which the functional forms of $b$ and $z^{i}$ is left intact, while the relationships between the constants - ie. charges/fluxes and parameters - is left undetermined. Then we procede to impose (2.7) and (2.9) to hopefully find new algebraic relationships between the constants. We have thus constructed a well-defined solution generating technique, which is applicable to a large class of extremal solutions where the above operations can be defined.

The set of deformations outlined above, when applied to extremal configurations, generally produces a non-extremal configuration. It must be noted that for all known solutions in the STU model, this deformation works algebraically, in the sense that one can always define an operation in parameter space that elevates the deformation to a symmetry of the system and hence the deformation generates a new-solution. However, in some cases, these solutions, though mathematically well-defined do not correspond to real physical backgrounds as they fail the standard tests of positivity of metric coefficients or finiteness of 4-derivative scalars constructed from the Riemann, Ricci tensor and the Ricci scalar. As a final step one has to check that the solutions obtained are physical.

Below, we give an illustrative example of how to implement the three-step deformation technique for a well-known magnetic extremal black brane [5] to produce a well defined non-extremal solution. This solution has a line element of the form (2.3) based on the prepotential (3.29). It is parametrized by 4 magnetic charges $P^{I}$ and four electric fluxes $h_{I}$. For extremal solution of this type, the hamiltonian constraint is given by [8]

$$
P^{I} h_{I}=0 .
$$

We can solve this constraint by taking

$$
P^{0}=-\frac{3}{16 h_{0}} \quad P^{i}=\frac{1}{16 h_{i}} .
$$

The warp factors for this solution read

$$
b^{2}=8 \prod_{I} \sqrt{\alpha^{I} r+\beta^{I}} \quad a b=\left(\gamma r^{2}+\delta\right),
$$


where

$$
\alpha^{I}=-\frac{\gamma}{4 h_{I}} \quad \beta^{I}=P^{I}
$$

$\gamma$ and $\delta$ are real constants subject to the constraint

$$
\gamma \delta=-\frac{3}{16} .
$$

Moreover, in order to avoid a naked singularity we must have $\gamma>0$ and the horizon is located at

$$
r_{H}=\frac{\sqrt{3}}{4 \gamma} .
$$

This solution can be found by making use of the first order equations $[3,8]$ for the prepotential (3.29). We now implement the three-step hot deformation:

Step 1: Deform the warp factors

$$
(a b)^{2} \rightarrow(a b)^{2}-\mu r
$$

keeping the functional forms of $b$ and $z^{i}$ fixed.

Step 2: Insert the deformation into the remaining equations and read off the corresponding algebraic equations. For the case at hand, these can be solved and they imply the modified relationships

$$
\begin{aligned}
P^{0} & =-\frac{3}{16 h_{0}} \longrightarrow P^{0}\left(1+\frac{48}{9} \mu \gamma\right)^{-1 / 2}=-\frac{3}{16 h_{0}} \\
P^{i} & =\frac{1}{16 h_{i}} \longrightarrow P^{i}(1-16 \mu \gamma)^{-1 / 2}=\frac{1}{16 h_{i}} .
\end{aligned}
$$

Step 3: We check that the solution is physical.

In summary, we have obtained a regular solution with

$$
b^{2}=8 \prod_{I} \sqrt{\alpha^{I} r+\beta^{I}} \quad(a b)^{2}=\left(\gamma r^{2}+\delta\right)^{2}-\mu r
$$

where

$$
\alpha^{I}=-\frac{\gamma}{4 h_{I}}
$$

and

$$
\beta^{0}=P^{0}\left(1+\frac{48}{9} \mu \gamma\right)^{-1 / 2} \quad \beta^{i}=P^{i}(1-16 \mu \gamma)^{-1 / 2} .
$$

This solution is non-extremal as it has two horizons, which for small $\mu$, are located at

$$
r_{ \pm}=r_{H} \pm \frac{\sqrt{\mu}}{3^{1 / 4}}+\ldots
$$

This technique should prove to be of tremendous utility in generating and studying hot solutions of relevance to holographic condensed matter and fluid dynamics, in the future. 


\section{Conclusions}

In this note we have developed new rewritings of $\mathcal{N}=24 D$ gSUGRA actions for a class of dyonic non-extremal and electric non-extremal solutions not contained in the dyonic class, obtained the non-extremal version of the Nernst brane in $A A d S_{5}$ and introduced a deformation technique applied on extremal solutions to generate new non-extremal ones. These techniques will be particularly useful in developing holographic black duals for computations in 3D CFTs dual to $A d S_{4}$ and Lifschitz geometries which can be obtained by choosing the flux quanta appropriately. A recent development in this field (see cf. [17]) has resulted in the existence of closed form solutions for the metric in asymptotically AdS spaces and where the scalars can be obtained by solving algebraic equations. The new classes of extremal solutions so obtained can now be plugged into the deformation toolkit we have introduced here to obtain whole new classes of non-extremal solutions. More excitingly, one should be able to easily extend this formalism to $5 D$ gauged supergravity where whole new extremal and non-extremal solution spaces could be uncovered with exciting implications for $4 D$ field theories. For example, based on past and recent research [15, 18, 19] and ongoing work by some of the authors [4], one can write down the affine parameter that characterizes the flow of scalar fields from boundary to the horizon of extremal black solutions and these are holographically dual to the c-function that parametrizes Wilsonian flow in the corresponding field theory. Hence one can make statements about the central charges of the UV and IR fixed points of the Wilsonian flow from trivial calculations in the bulk. Hence, the techniques developed here have easy extensions and applications which can impact not just the study of black holes but also holographically coded processes of field theories.

\section{Acknowledgments}

The authors are especially grateful to Gabriel Lopes Cardoso for invaluable discussion. We are also grafeful to Michele Cirafici, Michael Haack, Vishnu Jejjala, Giuseppe Policastro, Chiara Toldo and Jan Troost for stimulating conversations related to the work in this note. S.N. and A.V.O gratefully acknowledge support from the Center for Theoretical Physics at the University of the Witwaterstrand. S.N. is supported by a URC fellowship, an NRF grant and by a SARCHI fellowship. The work of A.V.O is supported in part by a URC fellowship. The work of KG is supported in part by the National Research Foundation. Any opinion, findings and conclusions or recommendations expressed in this material are those of the authors and therefore the NRF do not accept any liability with regard thereto.

\section{A Notation}

In this appendix we follow the notation found in [3], which for convenience is summarized below, and relate it to the parameters which appear in (2.1). The Lagrangian describing the couplings of $\mathcal{N}=2$ vector multiplets to $\mathcal{N}=2$ supergravity is encoded by a holomorphic function $F(X)$ called the prepotential, that depends on $n+1$ complex scalar fields $X^{I}$ 
$(I=0, \ldots, n) . F(X)$ is homogeneous of degree two, i.e. $F(\lambda X)=\lambda^{2} F(X)$, from which leads to the homogeneity properties

$$
\begin{aligned}
F_{I} & =F_{I J} X^{J}, \\
F_{I J K} X^{K} & =0,
\end{aligned}
$$

where $F_{I}=\partial F(X) / \partial X^{I}, F_{I J}=\partial^{2} F / \partial X^{I} \partial X^{J}$, etc. The $X^{I}$ are redundant while the physical scalar fields

$$
z^{i}=X^{i} / X^{0} \quad(i=1, \ldots, n)
$$

parametrize an $n$-dimensional complex hypersurface. The redundancy of the $X^{I}$ is encoded by a constraint on the symplectic vector $\left(X^{I}, F_{I}(X)\right)$ :

$$
i\left(\bar{X}^{I} F_{I}-\bar{F}_{I} X^{I}\right)=1 .
$$

This can be written as

$$
-N_{I J} X^{I} \bar{X}^{J}=1
$$

where

$$
N_{I J}=-i\left(F_{I J}-\bar{F}_{I J}\right) .
$$

For $\mathcal{N}=2$ based models, the lagrangian (2.1) is constructed out of

$$
g_{i \bar{\jmath}}=\frac{\partial^{2} K(z, \bar{z})}{\partial z^{i} \partial \bar{z}^{j}},
$$

where the Kähler potential is given by

$$
e^{-K}=i\left(\bar{X}^{I} F_{I}-X^{I} \bar{F}_{I}\right),
$$

as well as

$$
f_{I J}=\frac{1}{2} \operatorname{Im}\left(\mathcal{N}_{I J}\right) \quad \tilde{f}_{I J}=\frac{1}{2} \operatorname{Re}\left(\mathcal{N}_{I J}\right),
$$

where

$$
\mathcal{N}_{I J}=\bar{F}_{I J}+i \frac{N_{I K} X^{K} N_{J L} X^{L}}{X^{M} N_{M N} X^{N}} .
$$

and

$$
V_{g}=N^{I J} \hat{h}_{I} \overline{\hat{h}}_{J}-2 e^{K}|W|^{2},
$$

where

$$
\hat{h}_{I}=h_{I}-F_{I K} h^{K} \quad W=h^{I} F_{I}-h_{I} X^{I},
$$

and $\left(h^{I}, h_{I}\right)$ denote the magnetic and electric fluxes respectively — see [20] for a nice review. 


\section{B Explicit gauge field ansatz}

In this appendix we show our explicit gauge field ansatz for the three cases $k=-1,0,1$. To establish coordinates we take

$$
d \Omega_{k}^{2}= \begin{cases}d \theta^{2}+\sin ^{2} \theta d \phi^{2} & k=1 \\ d x^{2}+d y^{2} & k=0 \\ d \theta^{2}+\sinh ^{2} \theta d \phi^{2} & k=-1\end{cases}
$$

In these coordinates ansatze for the gauge fields are (cf. $[1,16])$

$$
A^{I}=\mathcal{Q}^{I} e^{-2 \beta} d t-P^{I} \begin{cases}\cos \theta d \phi & k=1 \\ x d y & k=0 \\ \cosh \theta d \phi & k=-1\end{cases}
$$

where

$$
\mathcal{Q}^{I}=f^{I J}\left(Q_{J}-\tilde{f}_{J K} P^{K}\right) .
$$

Using this ansatz the potential $V_{b}(2.8)$ can also be written

$$
V_{b}=N^{I J} \hat{Q}_{I} \overline{\hat{Q}}_{J}+2 e^{K}|Z|^{2},
$$

where

$$
\hat{Q}_{I}=Q_{I}-F_{I K} P^{K} \quad Z=P^{I} F_{I}-Q_{I} X^{I} .
$$

Open Access. This article is distributed under the terms of the Creative Commons Attribution License (CC-BY 4.0), which permits any use, distribution and reproduction in any medium, provided the original author(s) and source are credited.

\section{References}

[1] L. Andrianopoli et al., General matter coupled $N=2$ supergravity, Nucl. Phys. B 476 (1996) 397 [hep-th/9603004] [INSPIRE].

[2] S. Barisch-Dick, G. Lopes Cardoso, M. Haack and S. Nampuri, Extremal black brane solutions in five-dimensional gauged supergravity, JHEP 02 (2013) 103 [arXiv:1211.0832] [INSPIRE].

[3] S. Barisch, G. Lopes Cardoso, M. Haack, S. Nampuri and N.A. Obers, Nernst branes in gauged supergravity, JHEP 11 (2011) 090 [arXiv:1108.0296] [INSPIRE].

[4] A. Bhattacharya, S. Haque, V. Jejjala, S. Nampuri and A. Véliz-Osorio, Attractive holographic c-functions, arXiv:1407.0469 [INSPIRE].

[5] S.L. Cacciatori and D. Klemm, Supersymmetric AdS 4 black holes and attractors, JHEP 01 (2010) 085 [arXiv:0911.4926] [InSPIRE].

[6] G.L. Cardoso and V. Grass, On five-dimensional non-extremal charged black holes and FRW cosmology, Nucl. Phys. B 803 (2008) 209 [arXiv:0803.2819] [INSPIRE]. 
[7] A.H. Chamseddine and W.A. Sabra, Magnetic and dyonic black holes in D=4 gauged supergravity, Phys. Lett. B 485 (2000) 301 [hep-th/0003213] [INSPIRE].

[8] G. Dall'Agata and A. Gnecchi, Flow equations and attractors for black holes in $N=2 \mathrm{U}(1)$ gauged supergravity, JHEP 03 (2011) 037 [arXiv:1012.3756] [INSPIRE].

[9] B. de Wit and H. Nicolai, $N=8$ supergravity, Nucl. Phys. B 208 (1982) 323 [inSPIRE].

[10] F. Denef, Supergravity flows and D-brane stability, JHEP 08 (2000) 050 [hep-th/0005049] [INSPIRE].

[11] A. Donos, J.P. Gauntlett and C. Pantelidou, Semi-local quantum criticality in string/M-theory, JHEP 03 (2013) 103 [arXiv:1212.1462] [INSPIRE].

[12] S. Ferrara and R. Kallosh, Supersymmetry and attractors, Phys. Rev. D 54 (1996) 1514 [hep-th/9602136] [INSPIRE].

[13] A. Gnecchi and C. Toldo, First order flow for non-extremal AdS black holes and mass from holographic renormalization, arXiv:1406.0666 [INSPIRE].

[14] K. Goldstein, N. Iizuka, R.P. Jena and S.P. Trivedi, Non-supersymmetric attractors, Phys. Rev. D 72 (2005) 124021 [hep-th/0507096] [INSPIRE].

[15] K. Goldstein, R.P. Jena, G. Mandal and S.P. Trivedi, A C-function for non-supersymmetric attractors, JHEP 02 (2006) 053 [hep-th/0512138] [INSPIRE].

[16] N. Halmagyi, M. Petrini and A. Zaffaroni, BPS black holes in AdS $S_{4}$ from M-theory, JHEP 08 (2013) 124 [arXiv:1305.0730] [InSPIRE].

[17] S. Katmadas, Static BPS black holes in U(1) gauged supergravity, arXiv:1405.4901 [INSPIRE].

[18] R.C. Myers and A. Sinha, Seeing a c-theorem with holography, Phys. Rev. D 82 (2010) 046006 [arXiv: 1006.1263] [INSPIRE].

[19] R.C. Myers and A. Sinha, Holographic c-theorems in arbitrary dimensions, JHEP 01 (2011) 125 [arXiv: 1011.5819] [INSPIRE].

[20] H. Samtleben, Lectures on gauged supergravity and flux compactifications, Class. Quant. Grav. 25 (2008) 214002 [arXiv:0808.4076] [INSPIRE].

[21] P.K. Tripathy and S.P. Trivedi, Non-supersymmetric attractors in string theory, JHEP 03 (2006) 022 [hep-th/0511117] [INSPIRE]. 